\title{
Energetics of Mg and B adsorption on polar zinc oxide surfaces from first principles
}

\author{
Kazume Nishidate, * Masahito Yoshizawa, and Masayuki Hasegawa \\ Faculty of Engineering, Iwate University, Morioka 020-8551, Japan
}

(Received 26 July 2007; revised manuscript received 18 October 2007; published 28 January 2008)

\begin{abstract}
We have performed first-principles density-functional projector augmented-wave calculations to investigate the energetics of $\mathrm{Mg}$ and $\mathrm{B}$ adsorption on polar zinc oxide $\mathrm{ZnO})$ surfaces, thereby to understand the origin of the peculiar affinities between an epitaxial $\mathrm{MgB}_{2}$ film and the $\mathrm{ZnO}$ substrate. We found that the (0001) $\mathrm{Zn}$ surface is relatively inert, while the $(000 \overline{1}) \mathrm{O}$ surface is very reactive in the adsorption of atomic species, where a triplaner $\mathrm{BO}_{3}$ (a hump of $\mathrm{MgO}_{3}$ ) binding unit is formed as a consequence of the $\mathrm{B}(\mathrm{Mg})$ atom adsorption on the surface. These binding units would be formed at an early stage in the epitaxial growth of $\mathrm{MgB}_{2}$ film on the $\mathrm{ZnO}$ substrate, and subsequently lead to the formation of the reaction products that were recently found in the interface between the $\mathrm{MgB}_{2}$ film and the $\mathrm{ZnO}$ substrate.
\end{abstract}

DOI: 10.1103/PhysRevB.77.035330 PACS number(s): 73.20.Hb, 71.20.Nr, 71.55.Gs, 71.15.Mb

\section{INTRODUCTION}

The discovery of the superconductivity at a transition temperature $\left(T_{c}\right)$ of $39 \mathrm{~K}$ in magnesium diboride ${ }^{1}\left(\mathrm{MgB}_{2}\right)$ has attracted much attention for its scientific implications as well as technological applications. To fabricate $\mathrm{MgB}_{2}$-based superconductor devices, it is very important to use a highquality as-grown film prepared at low temperatures. An asgrown process without postannealing is desirable for developing devices such as tunneling junctions and multilayer structure of $\mathrm{MgB}_{2}$. Several groups have reported lowtemperature growth of $\mathrm{MgB}_{2}$ films on the $\mathrm{Al}_{2} \mathrm{O}_{3}(0001)$ or $\mathrm{Si}$ (111) substrates using molecular beam epitaxy (MBE), ${ }^{2-4}$ electron-beam evaporation, ${ }^{5}$ pulsed laser deposition, ${ }^{6}$ and sputtering. ${ }^{7}$ However, these substrates have rather large lattice mismatch with $\mathrm{MgB}_{2}(\delta \simeq 20 \%)$, which is unfavorable for the epitaxial growth. It has been reported that the use of in-plane-lattice buffer layers with little mismatch, such as AlN $(\delta \simeq 1.9 \%)$ and $\operatorname{TiZr}(\delta \simeq 3.6 \%)$, with $\mathrm{Al}_{2} \mathrm{O}_{3}(0001)$ substrate could improve the crystallinity of $\mathrm{MgB}_{2} \cdot{ }^{8,9}$ The $T_{c}$ values of $\mathrm{MgB}_{2}$ on the AlN and TiZr buffer layers were 29.8 and $35.1 \mathrm{~K}$, respectively. These results indicate the importance of minimizing lattice mismatch between layers. Recently, as-grown $\mathrm{MgB}_{2}$ thin films in an ultrahigh vacuum MBE cell have been fabricated under the conditions of low temperature and low growth rate. ${ }^{10}$ In these fabrications, several substrates such as $\mathrm{MgO}, \mathrm{ZnO}, \mathrm{Al}_{2} \mathrm{O}_{3}, \mathrm{SrTiO}_{3}$, and $\mathrm{Si}$ have been explored to further refine the crystallinity of $\mathrm{MgB}_{2}$ films. Among these substrates, $\mathrm{ZnO}$ has been thought to be the best as its lattice constant, $a=3.351 \AA$, is very close to that of the $\mathrm{MgB}_{2}, a=3.083 \AA$, and both have the same hexagonal lattice symmetry. In fact, $c$-axis oriented $\mathrm{MgB}_{2}$ film with $T_{c}=35.0 \mathrm{~K}$ on the polar $\mathrm{ZnO}$ substrate has been successfully fabricated, ${ }^{11}$ but, at the same time, a certain amount of unfavorable reaction products has also been found in the interface between the $\mathrm{MgB}_{2}$ film and the $\mathrm{ZnO}$ substrate. ${ }^{10}$ This experimental finding suggests the importance of the affinities between substrate and film in addition to minimizing lattice mismatch. Although these reaction products were supposed to be a derivative of the $\mathrm{Mg}$ adatom on the $\mathrm{ZnO}$ surface, its energetics, as well as the local atomic structure, has not yet been examined. In the present study, we use an $a b$ initio method to investigate the energetics of $\mathrm{Mg}$ and $\mathrm{B}$ adsorption on the $\mathrm{ZnO}$ polar surfaces, thereby to understand the origin of the peculiar affinities between the epitaxial $\mathrm{MgB}_{2}$ film and the $\mathrm{ZnO}$ substrate.

\section{COMPUTATIONAL DETAILS}

\section{A. Structural parameters of the $\mathrm{ZnO}$ crystal}

Density-functional electronic structure calculations are performed using the Vienna $a b$ initio simulation package (VASP). ${ }^{12}$ The total energy is calculated using the projector augmented-wave (PAW) method. ${ }^{13,14}$ We first determine the structural parameters of the $\mathrm{ZnO}$ crystal to verify the performance of our computational method. For this purpose, we adopt rather stringent computational conditions; the reciprocal space integration is performed with the $20^{3}$ dense Monkhorst-Pack mesh ${ }^{15}$ and the high cutoff energy of $500 \mathrm{eV}$ is used in the plane-wave expansion. The exchangecorrelation energy functional is treated in the generalizedgradient approximation (GGA) of Perdew et al. (GGA-PBE). ${ }^{16,17}$ For comparisons, we also adopt the local density approximation ${ }^{18,19}$ (LDA) and the GGA of Perdew and Wang (GGA-PW91). ${ }^{20}$ The lattice parameters optimized in the LDA are slightly underestimated as compared with the experimental values, while those in the GGA (PW91 and PBE) are slightly overestimated (Table I). A similar trend has

TABLE I. Optimized structural parameters of bulk $\mathrm{ZnO}$ and their comparisons with experiments. $a$ and $c$ are the basal and $c$-axis lattice constants of the wurtzite structure, and $u$ is the internal coordinate which determines the relative position of the $\mathrm{Zn}$ and $\mathrm{O}$ sublattice along the $c$ axis.

\begin{tabular}{lccc}
\hline \hline & $\begin{array}{c}a \\
(\AA)\end{array}$ & $\begin{array}{c}c \\
(\AA)\end{array}$ & $u$ \\
\hline LDA & 3.202 & 5.141 & 0.3817 \\
PW91 & 3.288 & 5.271 & 0.3817 \\
PBE & 3.295 & 5.282 & 0.3817 \\
Experimental $^{\mathrm{a}}$ & 3.250 & 5.207 & 0.3819 \\
\hline \hline
\end{tabular}

Reference 24. 


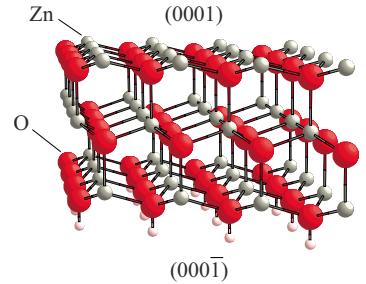

(a)

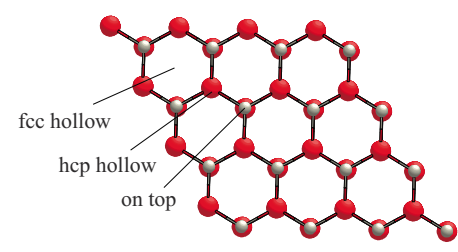

(b)
FIG. 1. (Color online) (a) Perspective and (b) top views of (0001) Zn surface slab. The large- and middle-sized spheres are the $\mathrm{O}$ and $\mathrm{Zn}$ atoms, respectively, and the small spheres below the bottom $(000 \overline{1})$ plane are hydrogenlike peseudoatoms introduced to saturate the dangling bonds. The geometry of the (0001) O surface (not shown here) is obtained by interchanging the atomic species, $\mathrm{Zn}$ and $\mathrm{O}$.

also been reported for $\mathrm{ZnO}$ crystal $^{21,22}$ and for a variety of materials including diatomic molecules. ${ }^{23}$ Hereafter, we adopt the GGA-PBE to keep consistency throughout this work.

\section{B. Surface slab}

$\mathrm{ZnO}$ is a wide-gap semiconductor and expected to be an important material for optoelectronic device applications. ${ }^{25}$ It consists of hexagonal $\mathrm{Zn}$ and $\mathrm{O}$ planes alternatively stacked along the $c$ axis. When the crystal is cleaved in the plane perpendicular to the $c$ axis, the two opposing polar surfaces are different; one is the (0001) $\mathrm{Zn}$ surface and the other the $(000 \overline{1}) \mathrm{O}$ surface. We may expect different reactivities for these two surfaces for the adsorption of $\mathrm{Mg}$ and $\mathrm{B}$ atoms. To investigate adsorption energetics of an isolated atom on the polar $\mathrm{ZnO}$ surfaces, we used a large hexagonal slab containing $4 \times 4 \times 2 \mathrm{ZnO}$ unit cells. Kresse et al. ${ }^{22}$ have suggested that the convergence of total energy calculations with respect to slab thickness can be accelerated by filling (removing) the electron holes (extra electrons) at the O-terminated ( $\mathrm{Zn}$ terminated) back side. We achieved convergence by saturating the dangling bonds on the back side of each polar surface; the oxygen (zinc) dangling bonds on the back side of (0001) Zn slab $[(000 \overline{1})$ O slab] are terminated by adding pseudoatoms with the valency of $0.5|e|(1.5|e|)$ [Fig. 1(a)]. The optimized bond lengths between a saturating pseudoatom and the $\mathrm{O}$ and $\mathrm{Zn}$ back side atoms are 1.056 and $1.623 \AA$, respectively. We considered three high-symmetry
TABLE II. Reference states and their optimized structural parameters used to evaluate the atomic chemical potentials $\mu_{X}$.

\begin{tabular}{|c|c|c|c|c|}
\hline & \multicolumn{2}{|c|}{ Reference state } & \multicolumn{2}{|c|}{ Structural parameter } \\
\hline & State & Symmetry & $\begin{array}{c}\text { Experimental } \\
(\AA)\end{array}$ & $\begin{array}{l}\text { Optimized } \\
(\AA)\end{array}$ \\
\hline \multirow[t]{2}{*}{$\mathrm{Mg}$} & Metal & hcp & $a=3.2093^{\mathrm{a}}$ & $a=3.1918$ \\
\hline & & & $c=5.2107$ & $c=5.1916$ \\
\hline \multirow[t]{2}{*}{$\mathrm{Zn}$} & Metal & hcp & $a=2.664^{\mathrm{a}}$ & $a=2.6570$ \\
\hline & & & $c=4.9494$ & $c=4.9343$ \\
\hline \multirow[t]{2}{*}{ B } & Metal & Rhombohedral & $a=5.06^{\mathrm{b}}$ & $a=5.041$ \\
\hline & & $R \overline{3} m$ & $\left(\alpha=58^{\circ} 4^{\prime c}\right)$ & $\left(\alpha=58^{\circ} 17^{\prime c}\right)$ \\
\hline $\mathrm{O}$ & ${ }^{3} \mathrm{O}$ & ${ }_{2}$ molecule $^{\mathrm{d}}$ & $r=1.208^{\mathrm{e}}$ & $r=1.2330\left(1.2203^{\mathrm{e}}\right)$ \\
\hline
\end{tabular}

${ }^{\mathrm{a}}$ Reference 26.

${ }^{\mathrm{b}}$ Reference 27.

${ }^{\mathrm{c}} \alpha$ is the angle between the crystallographic axes.

${ }^{\mathrm{d} O x y g e n}$ molecule in the spin triplet state. The atomic chemical potential is raised by $\sim 0.51 \mathrm{eV}$ if we adopt an oxygen molecule in the spin singlet state $\left({ }^{1} \mathrm{O}_{2}\right)$.

${ }^{\text {e}}$ Reference 23 .

points as the adsorption sites: ${ }^{21}$ "on top" position just above the surface atom, "hcp-hollow" above the atom in the second surface layer, and "fcc hollow" with no atom beneath [Fig. 1(b)]. A large vacuum region over the surface $(18.5 \AA)$ is taken to eliminate the interaction between the surfaces. We considered only two $k$ points in the slab calculations and the cutoff energy of $350 \mathrm{eV}$ was used for the plane-wave basis expansion because of the limited computational resources. All the atoms were allowed to relax under the condition that the residual force acting on each atom becomes less than $0.01 \mathrm{eV} / \AA$.

\section{Atomic chemical potentials}

The adsorption energy of an atom $X$ adsorbed on the site (=fcc, hcp, or top), $\Delta E_{\text {ads }}^{X_{\text {site }}}$, is defined here as

$$
\Delta E_{\text {ads }}^{X_{\text {site }}}=E_{\text {slab }+X_{\text {site }}}^{\mathrm{tot}}-E_{\text {slab }}^{\mathrm{tot}}-\mu_{X},
$$

where $E_{\text {slab }+X_{\text {site }}}^{\text {tot }}$ and $E_{\text {slab }}^{\text {tot }}$ are the total energies of the slab with and without an adatom $X$ on the site, respectively. The atomic chemical potentials $\mu_{X}$ of adatoms $X_{\text {site }}$ are evaluated separately in their reference states. Here, we define the

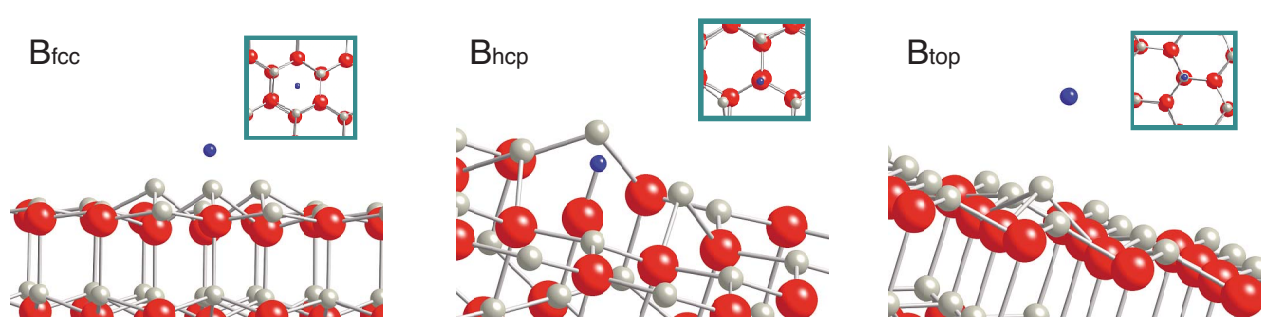

FIG. 2. (Color online) Atomic configuration of the (0001) Zn surface adsorbing a B atom. Top views are also shown in the squares. Large- and middle-sized spheres are the oxygen and zinc atoms, respectively, and the small dark spheres are the B adsorbed on the surface. 
TABLE III. Adsorption energies $\Delta E_{\text {ads }}$ (in unit of eV) and the local geometries of the adatom $X_{\text {site }}(X=\mathrm{B}$ or $\mathrm{Mg})$ on the site $(=\mathrm{fcc}$, hcp, or top) of the (0001) Zn surface. The numbers $(n)$ and species (spc.) of neighboring atoms within $3 \AA$ from the $X=$ site are tabulated. The distances between the adatom $X$ on the site and the neighboring atoms are indicated in parentheses (in unit of $\AA$ ).

\begin{tabular}{lcccc}
\hline \hline$X_{\text {site }}$ & & $\mathrm{fcc}$ & $\mathrm{hcp}$ & top \\
\hline $\mathrm{B}$ & $\Delta E_{\text {ads }}$ & 1.977 & 0.889 & 4.297 \\
& $n$ spc. & $3 \mathrm{Zn}(2.09)$ & $3 \mathrm{Zn}(2.19)$ & $\mathrm{Zn}(2.14)$ \\
& & $3 \mathrm{O}(2.93)$ & $\mathrm{O}(1.31)$ & $\cdots$ \\
$\mathrm{Mg}$ & $\Delta E_{\text {ads }}$ & 0.495 & 0.474 & 0.942 \\
& $n$ spc. & $2 \mathrm{Zn}(2.75)$ & $2 \mathrm{Zn}(2.71)$ & $\mathrm{Zn}(2.76)$ \\
& & $\mathrm{Zn}(2.77)$ & $\mathrm{Zn}(2.75)$ & $\cdots$ \\
\hline \hline
\end{tabular}

atomic chemical potential as a calculated total energy per atom in its optimized reference state. ${ }^{28}$ The adsorption energy defined in this way is not the same as the one observed in experiments but may be viewed as a conventional one accounting for the relative stability in the concerned adsorption process. Reciprocal space integrations were performed using the $20^{3}$ Monkhorst-Pack mesh ${ }^{15}$ for the bulk metals, and only the $\Gamma$ point is used for an $\mathrm{O}_{2}$ molecule placed in a large vacuum supercell with volume of $15^{3} \AA^{3}$. Converged results were obtained using the cutoff energy of $500 \mathrm{eV}$ for the plane-wave expansion. The reference states and their optimized structural parameters are summarized in Table II.

\section{Vacancy formation energies}

With the same computational details, we next evaluate the formation energy of a vacancy $V_{X}(X=\mathrm{Zn}$ or $\mathrm{O})$ on the surface defined as

$$
\Delta E_{f}^{V_{X}}=E_{\mathrm{slab}+V_{X}}^{\mathrm{tot}}-E_{\mathrm{slab}}^{\mathrm{tot}}+\mu_{X},
$$

where $E_{\mathrm{slab}+V_{X}}^{\mathrm{tot}}$ is the total energy of the slab with a vacancy on its surface. The equilibrium constraint for the atomic chemical potential ${ }^{29}$ is given by

$$
\mu_{\mathrm{ZnO}(\text { bulk })}=\mu_{\mathrm{Zn}}+\mu_{\mathrm{O}}+d h_{\mathrm{ZnO}(\text { bulk })} \text {, }
$$

where $\mu_{\mathrm{ZnO} \text { (bulk) }}$ is the chemical potential of bulk $\mathrm{ZnO}$ and customarily obtained as half of the total energy of the $\mathrm{ZnO}$ primitive unit cell. With this definition, we obtained enthalpy of formation for the $\mathrm{ZnO}$ crystal as $d h_{\mathrm{ZnO}(\text { bulk) }}=-2.903 \mathrm{eV}$,
TABLE IV. Adsorption energies $\Delta E_{\text {ads }}$ (in unit of $\mathrm{eV}$ ) of the adatom $X(\mathrm{~B}$ or $\mathrm{Mg})$ on the $(000 \overline{1}) \mathrm{O}$ surface. The other details are the same as those in Table III.

\begin{tabular}{ccccc}
\hline \hline & & fcc & hcp & top \\
\hline $\mathrm{B}$ & $\Delta E_{\text {ads }}$ & -5.822 & -5.642 & -0.115 \\
& $n$ atm. $(\AA)$ & $3 \mathrm{O}(1.40)$ & $\mathrm{O}(1.38)$ & $\mathrm{O}(1.27)$ \\
& & $3 \mathrm{Zn}(2.36)$ & $2 \mathrm{O}(1.42)$ & $3 \mathrm{Zn}(2.98)$ \\
& & $\mathrm{Zn}(2.35)$ & \\
$\mathrm{Mg}$ & $\Delta E_{\text {ads }}$ & -4.787 & -4.295 & -4.789 \\
& $n$ atm. $(\AA)$ & $3 \mathrm{O}(1.97)$ & $3 \mathrm{O}(2.00)$ & $3 \mathrm{O}(1.97)$ \\
& & $3 \mathrm{Zn}(2.67)$ & $\mathrm{Zn}(2.35)$ & $3 \mathrm{Zn}(2.67)$ \\
\hline \hline
\end{tabular}

which is compared to the experimental value of $-3.63 \mathrm{eV} .^{30}$ By adjusting the atomic chemical potentials in the range $\mu_{X}$ to $\mu_{X}+d h_{\mathrm{ZnO}}$, we can take into account the $\mathrm{Zn}$-rich and O-rich atmospheric conditions for the defect formations; i.e., in the Zn-rich (O-rich) condition, $\mu_{\mathrm{Zn}}\left(\mu_{\mathrm{O}}\right)$ is fixed at the value evaluated in its reference state, while $\mu_{\mathrm{O}}\left(\mu_{\mathrm{Zn}}\right)$ is replaced by $\mu_{\mathrm{O}}+d h_{\mathrm{ZnO}}\left(\mu_{\mathrm{Zn}}+d h_{\mathrm{ZnO}}\right)$. This procedure, which is simple and rather tractable, has been successfully used in the first-principles calculations to investigate the defect physics of semiconductors. ${ }^{29,31}$ Under the oxygen-rich condition, the formation energy of $V_{\mathrm{Zn}}$ on the (0001) $\mathrm{Zn}$ surface is $-3.42 \mathrm{eV}$ if we use the oxygen atomic chemical potential with respect to the oxygen molecule in its triplet state ${ }^{3} \mathrm{O}_{2}$ (see Table II). If the oxygen molecule in its singlet state ${ }^{2} \mathrm{O}_{2}$ is adopted as the reference state, that value becomes $-2.91 \mathrm{eV}$, which is compared with the reported theoretical value of $-3.11 \mathrm{eV}$ (extrapolated from Fig. 6 of Ref. 22). In the following, we use the $\mu_{\mathrm{O}}$ obtained in the ${ }^{3} \mathrm{O}_{2}$ state for consistency. For the oxygen vacancy concentration of $25 \%$, its formation energy per vacancy has been reported to be $+1.75 \mathrm{eV}$ for a sixfold $(2 \times 2)$ arrangement of the vacancy, $V_{\mathrm{O}}($ sixfold $)$, and $+1.54 \mathrm{eV}$ for a rectangular arrangement of the vacancy, $V_{\mathrm{O}}$ (rect). ${ }^{32}$ However, we obtained the formation energies of $+0.67 \mathrm{eV}$ for the $V_{\mathrm{O}}$ (sixfold) and $+0.46 \mathrm{eV}$ for the $V_{\mathrm{O}}(\mathrm{rect})$, both being about one-third of the previous ones. We cannot trace the origin of these numerical differences in detail but it is possibly ascribed to the difference of the models. In the previous calculations, ${ }^{32}$ the back side of the slab was not terminated and the extra energy income of the residual dipole would have contributed to the formation energies even after their own energy corrections. For our slab with only one vacancy (this is for the case with lower $V_{\mathrm{O}}$

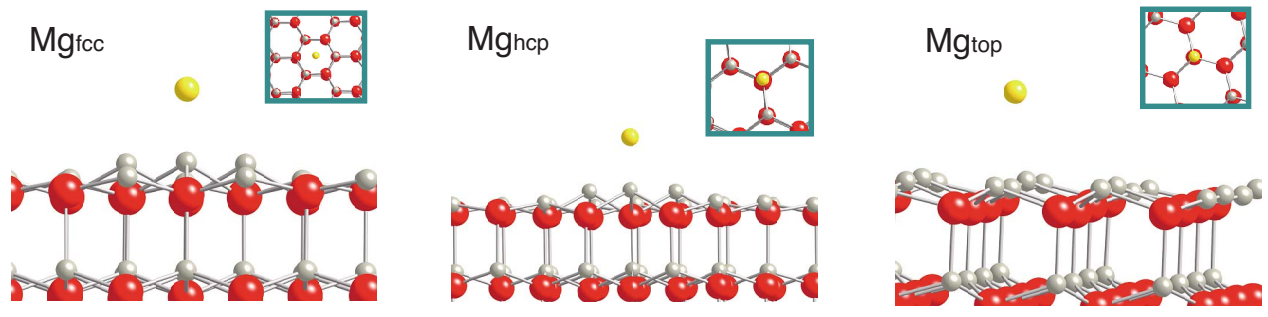

FIG. 3. (Color online) Atomic configuration of the (0001) Zn surface with an adsorbed Mg atom (middle-sized sphere). Top views are also shown in the squares. 

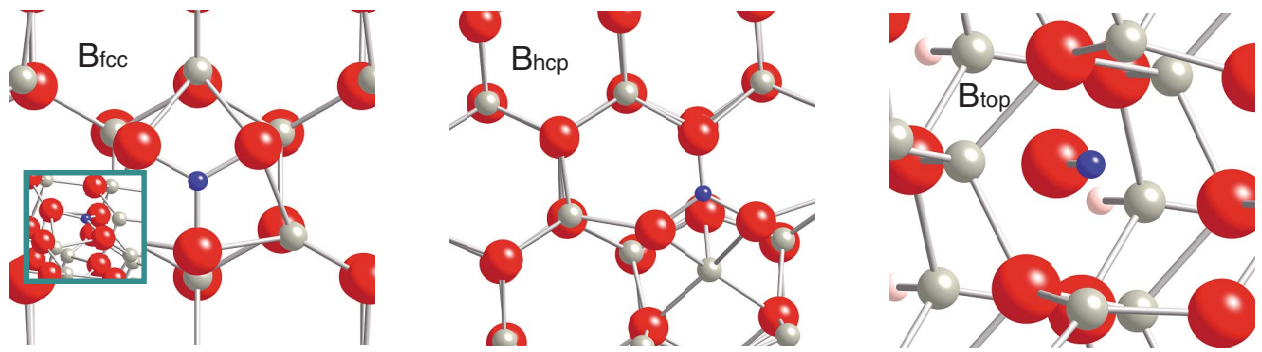

FIG. 4. (Color online) Atomic configuration of the (0001) O surface with an adsorbed B atom (small sphere). The view from a different angle is shown in the square.

concentration of $6.25 \%$ ), we obtained the formation energy of $+0.32 \mathrm{eV}$.

\section{RESULTS AND DISCUSSION}

\section{A. Adsorption on the (0001) Zn surface}

The results for the energetics of $\mathrm{B}$ and $\mathrm{Mg}$ adsorption on the (0001) Zn surface are summarized in Table III. Since the adsorption energies are obtained using the conventional atomic chemical potentials evaluated in their standard reference states, we cannot use those values for a quantitative comparison with experiments but may use them to examine the relative affinities of adatoms. The $\mathrm{B}$ atom adsorbed on the fcc site attracts three neighboring $\mathrm{Zn}$ atoms and lifts them from the surface (Fig. 2). Similarly, the B atom on the top site attracts one $\mathrm{Zn}$ atom from the surface. Both of these processes are unlikely to occur because of high energy cost (Table III). On the other hand, the B atom adsorbed on the hcp site forms a bond with the $\mathrm{O}$ atom just below the site with a bond length of $1.31 \AA$, thereby lowering the adsorption energy down to $0.89 \mathrm{eV}$. This result clearly indicates that the $\mathrm{B}$ atom preferably binds to the $\mathrm{O}$ atom rather than the $\mathrm{Zn}$ atom even on the (0001) Zn surface. All these adsorption processes of a B atom on the $\mathrm{Zn}$ surface are endothermic

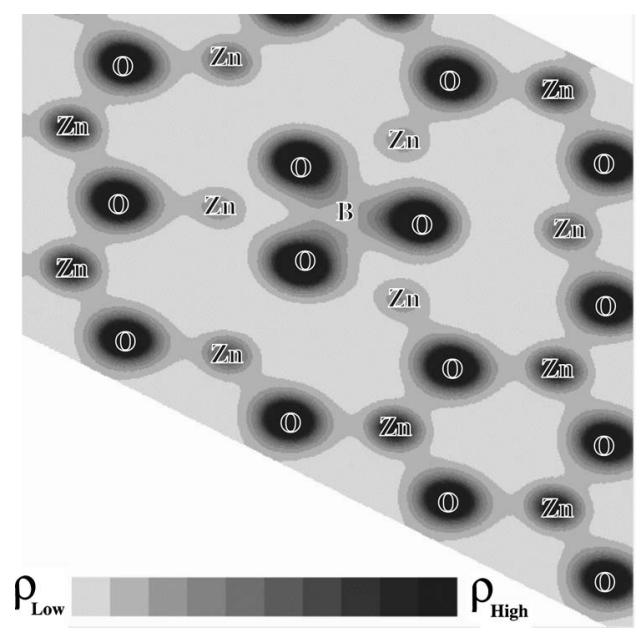

FIG. 5. Valence charge density $\rho(r)$ of the $(000 \overline{1})$ O surface with an adatom B on the fcc site. The region of high charge density $\rho_{\text {high }}$ is defined as that with $\rho_{\text {high }}>0.2 \rho_{\max }$, where the $\rho_{\max }$ is the maximum charge density. Lower densities are relative to $0.2 \rho_{\max }$. with respect to the reference atomic chemical potential. In contrast, the adsorption energies of the $\mathrm{Mg}$ atom on the fcc and hcp sites are almost the same and their local atomic configurations are also nearly identical (Fig. 3). Each $\mathrm{Mg}$ atom has three neighboring $\mathrm{Zn}$ atoms within the distance of $\sim 2.75 \AA$ (Table III). The adsorption of $\mathrm{Mg}$ on the top site needs about twice higher energy than in the other cases, implying that $\mathrm{Mg}$ is hardly adsorbed on the top site. Therefore, the $\mathrm{Mg}$ atom, as well as $\mathrm{B}$, on the (0001) $\mathrm{Zn}$ surface is unlikely to make strong bonds with the surface atoms and would show a rather diffusive behavior avoiding the route climbing over the $\mathrm{Zn}$ atom (top site).

\section{B. Adsorption on the $(000 \overline{1})$ O surface}

The results for the adsorption energetics and geometries of the $(000 \overline{1})$ O surface are summarized in Table IV. We find from these results that, in contrast to the $\mathrm{Zn}$ surface, this surface shows an extremely reactive behavior for the adsorption of $\mathrm{B}$ and $\mathrm{Mg}$ atoms.

The $\mathrm{B}$ atom on the fcc site forms triplaner bonds with the three neighbor $\mathrm{O}$ atoms in the distances of $\sim 1.4 \AA$ (Fig. 4). The valence charge density map of the $(000 \overline{1})$ surface clearly shows a covalent bonding feature of the triplaner $\mathrm{BO}_{3}$ binding unit (Fig. 5). Similarly, the B atom on the hcp site forms the same triplaner local atomic configuration by driving out the $\mathrm{Zn}$ atom just beneath. Figure 6 schematically shows the triplaner $\mathrm{BO}_{3}$ binding unit found at these adsorption sites. The adsorption energies of these processes are quite low, and thus all energetically favorable. We note here that the triplaner binding unit is an elemental framework in the $\mathrm{B}_{2} \mathrm{O}_{3}$

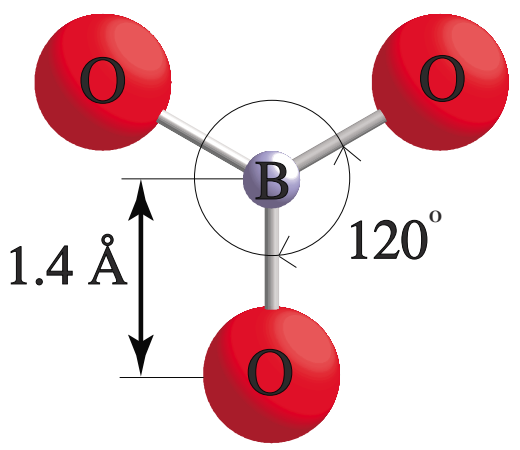

FIG. 6. (Color online) The triplaner $\mathrm{BO}_{3}$ binding unit found at the fcc and hep sites on the $(000 \overline{1}) \mathrm{O}$ surface. 

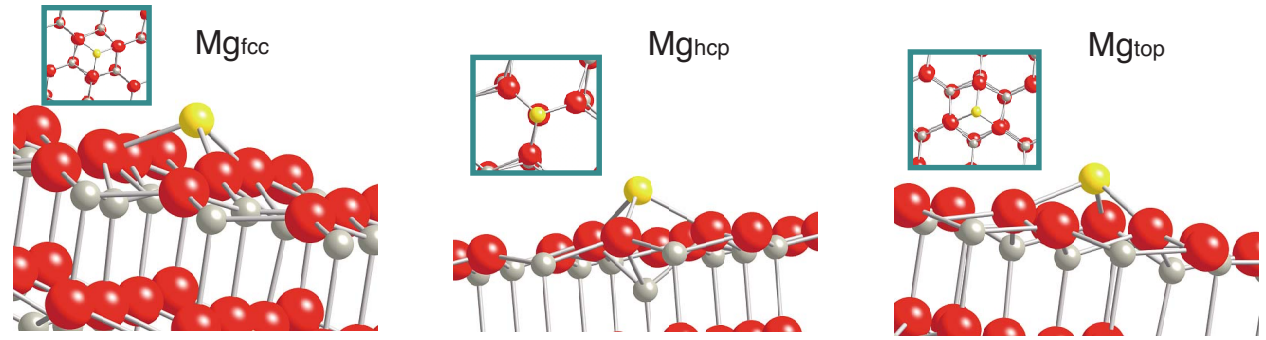

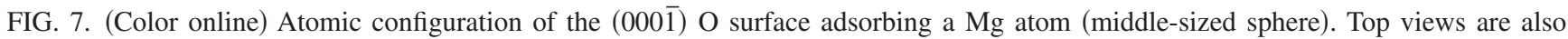
shown in the squares.

crystal, ${ }^{33}$ where the B-O bond length takes the same value of $\sim 1.4 \AA$. The $\mathrm{B}$ atom on the top site binds only to the nearest $\mathrm{O}$ atom with the distance of $1.27 \AA$ and the adsorption energy is still negative. Therefore, the adsorption on the top site is exothermic but would be unfavorable because of its relatively high energy cost $(\geqslant+5.5 \mathrm{eV})$ compared to the adsorptions on the fcc and hep sites.

The configurations of $\mathrm{Mg}$ atoms adsorbed on the fcc, hcp, and top sites of the $\mathrm{O}$ surface show similar local structures. Each of the $\mathrm{Mg}$ atoms binds to the three neighbor $\mathrm{O}$ atoms at the distance of $\sim 2 \AA$ (Fig. 7). In this case, the $\mathrm{O}$ atoms are lifted from the surface and forms a hump of $\mathrm{MgO}_{3}$ binding unit on the surface. The $\mathrm{O}-\mathrm{Mg}-\mathrm{O}$ bond angle of the binding unit is $\sim 100^{\circ}$. We also find that the $\mathrm{Mg}$ atom on the hep site drives the nearest $\mathrm{Zn}$ atom out of the original position below the $\mathrm{Mg}$ atom. Hence, it is energetically favorable for the $\mathrm{Mg}$ atom to be adsorbed on that site even though it induces deformation of the existing $\mathrm{Zn}-\mathrm{O}$ bond. We can see a similar local atomic structure in the $\mathrm{MgO}$ crystal, ${ }^{34}$ where each $\mathrm{Mg}$ atom has six neighbor $\mathrm{O}$ ligands at the distance of $\sim 2.1 \AA$ and a $\mathrm{MgO}_{6}$ cage is formed, whereas the $\mathrm{Mg}$ atom on the $(000 \overline{1}) \mathrm{O}$ surface has only three neighbor $\mathrm{O}$ atoms at shorter distances of 1.97-2.0 $\AA$ and their local geometric structure can be viewed as a fraction of a deformed $\mathrm{MgO}_{6}$ cage in the $\mathrm{MgO}$ crystal.

\section{Migration energetics on the (0001) Zn surface}

We use the well-established climbing nudged-elastic-band (C-NEB) technique ${ }^{35,36}$ to explore the energy profiles for the migration process of the adatoms, $\mathrm{Mg}$ and $\mathrm{B}$, on the (0001) $\mathrm{Zn}$ surface. The calculated energy profile for the Mg migration along the $\mathrm{fcc} \rightarrow \mathrm{hcp} \rightarrow$ top $\rightarrow$ fcc path is shown in Fig. 8 . The energy barrier for the $\mathrm{Mg}$ migration from the fcc site to the hcp site is only $0.06 \mathrm{eV}$. On the other hand, the barrier for the $\mathrm{Mg}$ migration along the path climbing over the top site amounts to $\sim 0.5 \mathrm{eV}$. Therefore, the adsorbed $\mathrm{Mg}$ atom would migrate along the path with the lowest energy barrier, $\mathrm{fcc} \rightarrow$ hcp. We note that it is possible for the $\mathrm{Mg}$ to migrate throughout the surface area by taking a zigzag route (fcc $\rightarrow$ hcp $\rightarrow$ fcc $\rightarrow \cdots)$, i.e., the $\mathrm{Mg}$ atom adsorbed on the (0001) $\mathrm{Zn}$ surface shows high mobility characteristics. On the other hand, the energy profile for the B migration on the (0001) Zn surface shows a quite different behavior (Fig. 9). The energy barrier for the B migration for the $\mathrm{fcc} \rightarrow$ hcp route amounts to $\sim 1.4 \mathrm{eV}$, while it is $\sim 2.4 \mathrm{eV}$ for the reverse route. There is a maximum energy barrier $(\sim 3.8 \mathrm{eV})$ for the $\mathrm{B}$ migration along the hcp $\rightarrow$ top path. The migration of B along the fcc $\rightarrow$ top path also encounters a high energy cost $(\sim 2.8 \mathrm{eV})$. Therefore, neither of these migration processes would be possible and the B atom adsorbed on the fcc and hep sites is rather immovable on the (0001) $\mathrm{Zn}$ surface and could make bonds with $\mathrm{O}$ atoms. We also tried to calculate the energy

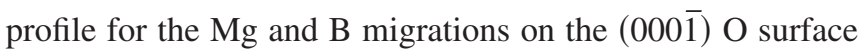
but could not obtain any converged result. The tendency of divergence in the $\mathrm{C}$-NEB computation of $\mathrm{O}$ surface is understandable since the adatoms form strong bonds with surface $\mathrm{O}$ atoms. Any of the intermediate migration point between the specific adsorption sites, and hence the migration path on the $\mathrm{O}$ surface, would be indefinite.

Kresse et al. ${ }^{22}$ have reported that there is a unique stabilization mechanism in the (0001) Zn polar surface, where a certain amount of triangular-shaped defects (pits) are formed to effectively maintain its valency, implying that some oxy-

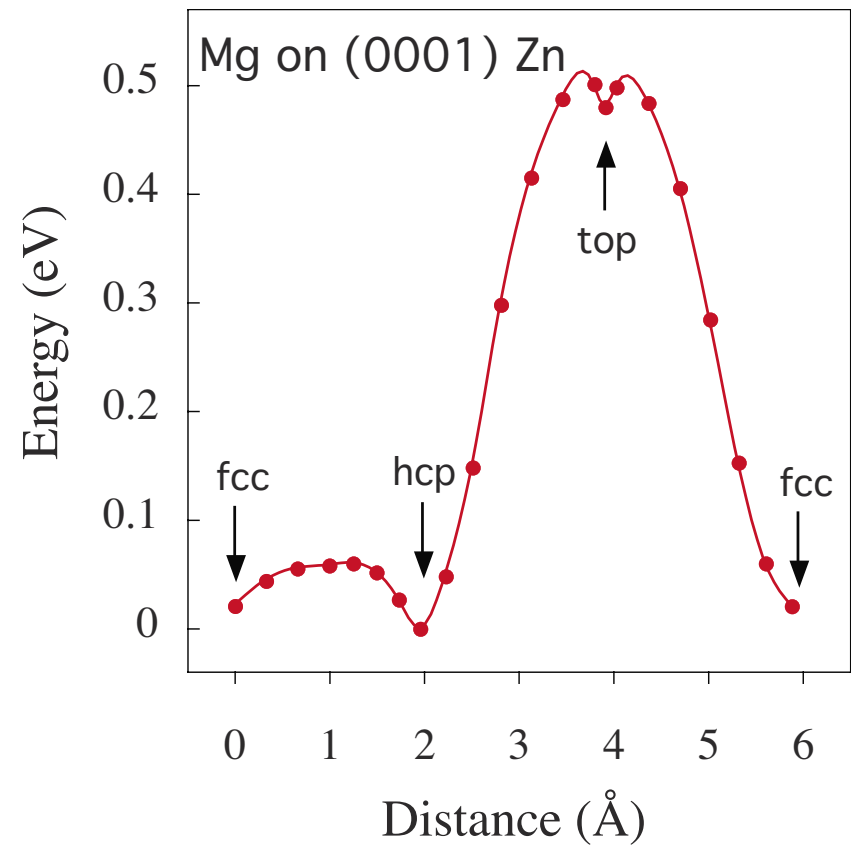

FIG. 8. (Color online) Energy profile for the Mg migration along the fcc $\rightarrow$ hcp $\rightarrow$ top $\rightarrow$ fcc path on the (0001) Zn surface. The energies are measured from the most stable state (hcp site). The horizontal axis is the integrated distance along the optimized migration path. The circles are calculated points and the curve is a guide for the eye. 


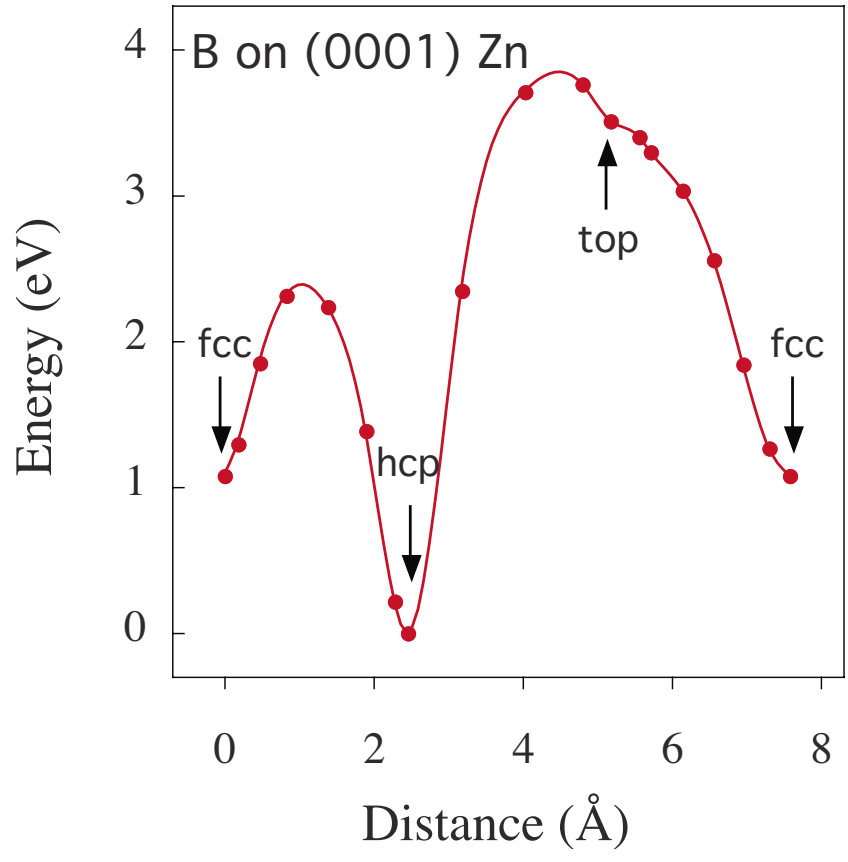

FIG. 9. (Color online) Energy profile for the B migration along the $\mathrm{fcc} \rightarrow \mathrm{hcp} \rightarrow$ top $\rightarrow$ fcc path on the (0001) Zn surface. The other details are the same as those in Fig. 8.

gen atoms are bare at these intrinsic pits even on the clean (0001) Zn surface. Our results indicate that the bare oxygen atoms, not only on the $(000 \overline{1}) \mathrm{O}$ surface but also on the (0001) Zn surface, easily make bonds with both $\mathrm{Mg}$ and B atoms. These reactions will continue until the excess oxygen atoms are exhausted on the surface, and as a result, a thin layer of the reaction products will be left behind in the interface between the epitaxial $\mathrm{MgB}_{2}$ film and the $\mathrm{ZnO}$ substrate.

\section{CONCLUSIONS}

A first-principles density-functional PAW method was used to investigate the energetics of $\mathrm{Mg}$ and $\mathrm{B}$ adsorption on the polar $\mathrm{ZnO}$ surfaces, thereby to understand the origin of the peculiar affinities between the epitaxial $\mathrm{MgB}_{2}$ film and the $\mathrm{ZnO}$ substrate. We found that the (0001) $\mathrm{Zn}$ surface is relatively inert with respect to the $\mathrm{Mg}$ and $\mathrm{B}$ adatoms. On that surface, only the B atom on the hep site forms a bond with the $\mathrm{O}$ atom just below the surface $\mathrm{Zn}$ layer and its adsorption energy is as low as $0.89 \mathrm{eV}$, which clearly indicates that the $\mathrm{B}$ atom favors to bind to the $\mathrm{O}$ atom rather than to the $\mathrm{Zn}$ atom even on the (0001) Zn surface. Therefore, both $\mathrm{Mg}$ and $\mathrm{B}$ atoms are unlikely to make strong bonds with the surface $\mathrm{Zn}$ atoms and would show rather diffusive behavior avoiding the route climbing over the $\mathrm{Zn}$ atom (top site). On the other hand, the B atoms adsorbed on both the fcc and hcp sites of the $(000 \overline{1}) \mathrm{O}$ surface form the triplaner $\mathrm{BO}_{3}$ binding units, which can also be found in the $\mathrm{B}_{2} \mathrm{O}_{3}$ crystal. The $\mathrm{Mg}$ atoms adsorbed on the fcc and hcp sites of that surface form a hump of $\mathrm{MgO}_{3}$ binding unit that can be viewed as a fraction of a $\mathrm{MgO}_{6}$ cage found in the $\mathrm{MgO}$ crystal. These results suggest that the bare oxygen atoms on the $\mathrm{ZnO}$ surface easily make bonds with both the $\mathrm{Mg}$ and $\mathrm{B}$ atoms. These reactions will continue until the excess oxygen atoms are exhausted on the surface and the binding units will be left behind. We note here that a certain amount of triangular-shaped pits are formed even on the clean $\mathrm{Zn}$ surface to reduce its valency. ${ }^{22}$ In these pits, some oxygen atoms are bared and again make bonds with both $\mathrm{Mg}$ and $\mathrm{B}$ atoms. As the result, the surface reactions will continue until the excess oxygen atoms are exhausted on both surfaces, leading to the formation of the reaction products that were recently found in the interface between the $\mathrm{MgB}_{2}$ film and the $\mathrm{ZnO}$ substrate. These theoretical results imply that the use of an appropriate buffer layer, which effectively covers the surface oxygen atoms, could suppress the formation of reaction products in the interfaces, or, alternatively, some gettering process for the oxygen at an initial stage of the film formation could work to prevent the formation of reaction products. We need more detailed experimental data for the $\mathrm{Mg}$ and $\mathrm{B}$ adsorption on the polar $\mathrm{ZnO}$ surfaces to verify these implications.

\section{ACKNOWLEDGMENTS}

The computations in the present work were performed using the facilities of the Institute for Solid State Physics, University of Tokyo, and of the Information Processing Center of Iwate University. We are grateful to G. Henkelman for providing the $\mathrm{C}$-NEB code. We are also grateful to $\mathrm{Y}$. Komatsu and $\mathrm{K}$. Ohta for assistance in the computations. K.N. thanks M. Baba and Y. Harada for valuable discussions.

\footnotetext{
*nisidate@iwate-u.ac.jp

${ }^{1}$ J. Nagamatsu, N. Nakagawa, T. Muranaka, Zenitani, and J. Akimitsu, Nature (London) 410, 63 (2001).

${ }^{2}$ K. Ueda and M. Naito, J. Appl. Phys. 93, 2113 (2003).

${ }^{3}$ W. Jo, J.-H. Huh, T. Ohnishi, A. Marshall, M. Beasley, and R. Hammond, Appl. Phys. Lett. 80, 3563 (2002).

${ }^{4}$ M. A. J. van Erven, T. H. Kim, and J. Moodera, Appl. Phys. Lett. 81, 4982 (2002).

${ }^{5}$ H. Kitaguchi, A. Matsumoto, H. Kumakura, T. Doi, H. Yamamoto, K. Saito, and S. Hata, Appl. Phys. Lett. 85, 2842 (2004).
}

${ }^{6}$ G. Grassano et al., Supercond. Sci. Technol. 14, 762 (2001).

${ }^{7}$ A. Saito, A. Kawakami, H. Shimakage, and Z. Wang, Jpn. J. Appl. Phys., Part 2 41, L127 (2002).

${ }^{8}$ K. Tsujimoto, H. Shimakage, Z. Wang, and N. Kaya, Physica C 426-431, 1464 (2005).

${ }^{9}$ O. Sakata, S. Kimura, M. Tanaka, S. Yata, T. Kato, K. Yamamoto, Y. Yamada, A. Matsushita, and S. Kubo, J. Appl. Phys. 96, 3580 (2004).

${ }^{10}$ T. Takahashi, Y. Harada, H. Iriuda, M. Kuroha, T. Oba, M. Seki, Y. Nakanishi, J. Echigoya, and M. Yoshizawa, Physica C 445- 
448, 887 (2006).

${ }^{11}$ Y. Harada, T. Takahashi, M. Kuroha, H. Iriuda, Y. Nakanishi, F. Izumida, H. Endo, and M. Yoshizawa, Physica C 445-448, 884 (2006).

${ }^{12}$ G. Kresse and J. Furthmüller, Phys. Rev. B 54, 11169 (1996), and references therein.

${ }^{13}$ G. Kresse and D. Joubert, Phys. Rev. B 59, 1758 (1999).

${ }^{14}$ P. E. Blöchl, Phys. Rev. B 50, 17953 (1994).

${ }^{15}$ H. J. Monkhorst and J. D. Pack, Phys. Rev. B 13, 5188 (1976).

${ }^{16}$ J. P. Perdew, K. Burke, and M. Ernzerhof, Phys. Rev. Lett. 77, 3865 (1996).

${ }^{17}$ J. P. Perdew, K. Burke, and M. Ernzerhof, Phys. Rev. Lett. 78, 1396 (1997).

${ }^{18}$ D. M. Ceperley and B. J. Alder, Phys. Rev. Lett. 45, 566 (1980).

${ }^{19}$ J. P. Perdew and A. Zunger, Phys. Rev. B 23, 5048 (1981).

${ }^{20}$ J. P. Perdew and Y. Wang, Phys. Rev. B 45, 13244 (1992).

${ }^{21}$ B. Meyer and D. Marx, Phys. Rev. B 69, 235420 (2004).

${ }^{22}$ G. Kresse, O. Dulub, and U. Diebold, Phys. Rev. B 68, 245409 (2003).

${ }^{23}$ D. C. Patton, D. V. Porezag, and M. R. Pederson, Phys. Rev. B 55, 7454 (1997).

${ }^{24}$ J. Albertsson, S. C. Abrahams, and Å. Kvick, Acta Crystallogr., Sect. B: Struct. Sci. 45, 34 (1989).

${ }^{25}$ Ü. Özgür, Ya. I. Alivov, C. Liu, A. Teke, M. A. Reshchikov, S.
Doğan, V. Avrutin, S.-J. Cho, and H. Morkoç, J. Appl. Phys. 98, 041301 (2005).

${ }^{26}$ A. Nayeb-Hashemi and J. Clark, Phase Diagrams of Binary Magnesium Alloys, Monograph Series on Alloy Phase Diagrams (ASM International, Metals Park, OH, 1988).

${ }^{27}$ L. McCarty, J. Kasper, F. Horn, B. Decker, and A. Newkirk, J. Am. Chem. Soc. 80, 2592 (1958).

${ }^{28}$ K. Reuter and M. Scheffler, Phys. Rev. B 65, 035406 (2001).

${ }^{29}$ K. Nishidate, T. Sato, Y. Matsukura, M. Baba, M. Hasegawa, and T. Sasaki, Phys. Rev. B 74, 035210 (2006), and references therein.

${ }^{30}$ CRC Handbook of Chemistry and Physics, 82nd ed. (CRC, New York, 2001).

${ }^{31}$ C. G. Van de Walle and J. Neugebauer, J. Appl. Phys. 95, 3851 (2004).

${ }^{32}$ B. Meyer, Phys. Rev. B 69, 045416 (2004).

${ }^{33}$ G. E. Gurr, P. W. Montgomery, C. D. Knutson, and B. T. Gorres, Acta Crystallogr., Sect. B: Struct. Crystallogr. Cryst. Chem. 26, 906 (1970).

${ }^{34}$ C. B. Walker and M. Marezio, Acta Metall. 7, 769 (1959).

${ }^{35}$ G. Henkelman, B. Uberuaga, and H. Jónsson, J. Chem. Phys. 113, 9901 (2000).

${ }^{36}$ G. Henkelman and H. Jónsson, J. Chem. Phys. 113, 9978 (2000). 